\title{
A 3' UTR sequence stabilizes termination codons in the unspliced RNA of Rous sarcoma virus
}

\author{
JASON E. WEIL and KAREN L. BEEMON \\ Department of Biology, The Johns Hopkins University, Baltimore, Maryland 21218, USA
}

\begin{abstract}
Eukaryotic cells target mRNAs to the nonsense-mediated mRNA decay (NMD) pathway when translation terminates within the coding region. In mammalian cells, this is presumably due to a downstream signal deposited during pre-mRNA splicing. In contrast, unspliced retroviral RNA undergoes NMD in chicken cells when premature termination codons (PTCs) are present in the gag gene. Surprisingly, deletion of a 401-nt $3^{\prime}$ UTR sequence immediately downstream of the normal gag termination codon caused this termination event to be recognized as premature. We termed this $3^{\prime}$ UTR region the Rous sarcoma virus (RSV) stability element (RSE). The RSE also stabilized the viral RNA when placed immediately downstream of a PTC in the gag gene. Deletion analysis of the RSE indicated a smaller functional element. We conclude that this $3^{\prime}$ UTR sequence stabilizes termination codons in the RSV RNA, and termination codons not associated with such an RSE sequence undergo NMD.
\end{abstract}

Keywords: NMD; RNA surveillance; translation termination; Rous sarcoma virus

\section{INTRODUCTION}

Eukaryotic cells employ a quality control mechanism to eliminate mRNAs bearing premature termination codons (PTC) (Hilleren and Parker 1999; Gonzalez et al. 2001; Maquat 2004; Wilkinson 2005). This mechanism of posttranscriptional control may have evolved not only to eliminate aberrant mRNAs, but also to regulate the level of some mRNAs (Lewis et al. 2003; Neu-Yilik et al. 2004). In mammalian cells, the nonsense-mediated mRNA decay (NMD) machinery recognizes a termination codon as premature if it is more than 50 nucleotides (nt) upstream of the last exon-exon boundary (Zhang et al. 1998a; Sun et al. 2000). During splicing, an exon junction complex (EJC) is deposited on the RNA 20-24 nt upstream of splice junctions (Le Hir et al. 2000a). This complex includes RNA splicing, export, cytoplasmic localization, and NMD factors (Le Hir et al. 2000a,b, 2001; Lykke-Andersen et al. 2000; Kim et al. 2001a,b; Chan et al. 2004). When a termination codon is encountered, the terminating ribosome and its associated factors recognize a downstream EJC through interactions with the Upf proteins, and the RNA is targeted for rapid destruction by the NMD machinery (Lykke-Andersen et al.

Reprint requests to: Karen L. Beemon, Ph.D, Department of Biology, Johns Hopkins University, 3400 North Charles Street, Baltimore, MD 21218, USA; e-mail: KLB@jhu.edu; fax: (410) 516-7292; phone: (410) 516-7289.

Article published online ahead of print. Article and publication date are at http://www.rnajournal.org/cgi/doi/10.1261/rna.2129806.
2000; He and Jacobson 2001; Lykke-Andersen 2002). In turn, naturally intronless genes are immune to NMD in mammalian cells (Maquat and Li 2001; Brocke et al. 2002).

Conversely, in yeast very few genes contain introns (Lopez and Seraphin 2000), and these unspliced mRNAs readily undergo NMD in a Upf-dependent fashion (Hilleren and Parker 1999; Gonzalez et al. 2001). This decay appears to be directed either by a coding region destabilizing element or distance from the poly $(\mathrm{A})$ tail (for review, see Hilleren and Parker 1999). Recent experiments in which the poly(A) binding protein was tethered downstream of a PTC resulted in stabilization of the NMD substrate (Amrani et al. 2004).

In contrast to the paradigm in mammalian cells that NMD is linked to splicing, Rous sarcoma virus (RSV) RNA undergoes NMD in chicken cells in the absence of splicing (Barker and Beemon 1991, 1994; LeBlanc and Beemon 2004). The 9.3-kb unspliced viral RNA is exported to the cytoplasm where it serves as both the genome of progeny virions and the mRNA template for the Gag and the Gag-Pol polyproteins (Swanstrom and Wills 1997). In most cases, only the first gene, gag, is translated, resulting in a 7$\mathrm{kb} \mathrm{3}$ ' untranslated region (UTR) (Fig. 1A). Approximately $5 \%$ of the time, a -1 programmed ribosomal frameshift moves the ribosome into the reading frame of the second viral gene, pol, resulting in synthesis of a Gag-Pol fusion protein (Swanstrom and Wills 1997). Barker and Beemon (1991, 1994) reported that premature termination codons (PTCs) in the gag gene lead to a substantial decrease in unspliced cytoplasmic viral RNA. This decay diminishes 

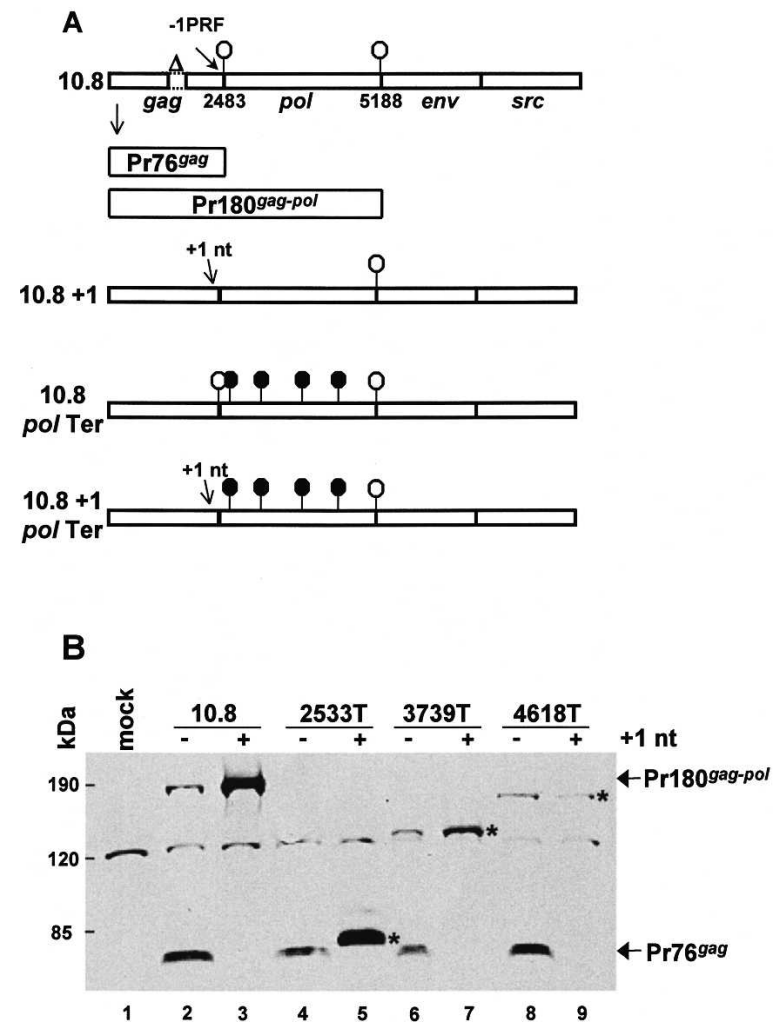

FIGURE 1. A 1-nucleotide (nt) insertion before the gag termination codon forces readthrough to the pol gene. $(A)$ The 10.8 plasmid contains a full-length copy of the Prague C strain of RSV with a deletion in the nucleocapsid region of the gag gene $(\Delta)$. All viral clones in this study originated from the 10.8 background. The gag gene is translated to generate a $76-\mathrm{kDa}$ precursor protein $\left(\operatorname{Pr} 76^{\text {gag }}\right)$. A -1 programmed ribosomal frameshift ( $-1 \mathrm{PRF}$ ) leads to the synthesis of a $180 \mathrm{kDa}$ Gag-Pol fusion polypeptide (Pr180 gag-pol $) .10 .8+1$ has a 1-nt insertion at position 2482, putting gag and pol in the same reading frame. $10.8 \mathrm{pol}$ Ter constructs have a PTC in the pol gene, and $10.8+1$ pol Ter constructs have the same PTC in the $10.8+1$ background. The white stop signs designate the gag and pol natural termination codons. The black stop signs represent PTCs in pol. (B) Western blot of CEFs transiently transfected with $10.8 \mathrm{pol}$ Ter and $10.8+1 \mathrm{pol}$ Ter constructs. The position of the termination codon in pol is given in nt (i.e., 2533T). The intermediate sized bands (denoted by ${ }^{*}$ ) in lanes 5,7 , and 9 are truncated Gag-Pol polypeptides due to the inserted termination codon. Viral proteins were detected using an antibody against AMV p19 (Matrix).

gradually when the PTC is in the final $150 \mathrm{nt}$ of the gag gene, approaching the natural termination codon (Barker and Beemon 1994). Additionally, LeBlanc and Beemon (2004) showed that this decay depends on translation and the critical NMD factor Upf1, implicating the NMD machinery. Since this regulation occurs on an unspliced RNA, it contrasts with previous reports in higher eukaryotes that splicing is necessary to distinguish a PTC from a normal termination codon (Maquat 2004).

In this report, we have characterized a region of the RSV genome found after the gag termination codon, which stabilizes the full-length unspliced RNA in chicken embryo fibroblasts (CEFs). We have termed this region the RSV stability element (RSE). We uncovered this element when examining termination codons in the pol gene. We observed that PTCs 500 nt or more into the pol gene only caused a lower steady state level of RNA when pol was placed in the same reading frame as the gag gene. Conversely, a PTC at the beginning of pol (within the RSE) did not result in a lower accumulation of RNA. Interestingly, a deletion downstream of the normal gag termination codon (the RSE region) led to a lower accumulation of unspliced RNA. This instability required translation and the protein Upf1, suggesting that deletion of the RSE leads to recognition of the gag natural termination codon as premature and induces NMD. In turn, insertion of the RSE immediately downstream of a PTC in gag stabilized the RNA. We conclude that there is a stability element found after the gag termination codon in RSV, and this region can stabilize an immediately preceding termination event.

\section{RESULTS}

\section{Translation of PTCs in pol destabilize the unspliced RNA}

Previous results have shown that termination codons placed throughout the gag coding region destabilize the unspliced RSV transcript (Barker and Beemon 1991) by the NMD pathway (LeBlanc and Beemon 2004). Knowing that the pol gene is translated much less frequently than gag (Swanstrom and Wills 1997), we asked whether termination codons in pol would cause degradation of the unspliced RNA.

Toward this end, we used the 10.8 viral construct, a clone of the Prague C strain of RSV, harboring a deletion in the nucleocapsid region of the gag gene (Meric et al. 1988). This deletion prevents packaging of the viral unspliced RNA. Barker and Beemon (1994) reported that a termination codon placed early in the pol gene (nt 2598) had no substantial effect on unspliced RNA stability. However, this could be due to the infrequent -1 frameshift, which leads to infrequent translation of the pol gene. To address the question of whether engineered termination codons throughout pol cause NMD, we constructed the plasmid $10.8+1 ; 10.8+1$ contains a single adenine insertion at nt 2482, immediately upstream of the gag natural termination codon. This insertion places gag and pol in the same reading frame, ensuring efficient translation of the Gag-Pol fusion polypeptide (Fig. 1A). We then made a series of constructs, each possessing a termination codon within the pol gene, in either the 10.8 or the $10.8+1$ background (Fig. 1A). CEF cells were transiently transfected with the above constructs, and 2-d post-transfection protein and RNA were harvested.

A Western blot using anti-p19 (Matrix) antibody to detect RSV Gag proteins showed that the majority of protein translated from the 10.8 transcript was the Gag protein, $\operatorname{Pr} 76^{\text {gag }}$, with a smaller amount of $\operatorname{Pr} 180^{\text {gag-pol }}$ readthrough product (Fig. 1B, lane 2). In contrast, translation of $10.8+1$ 


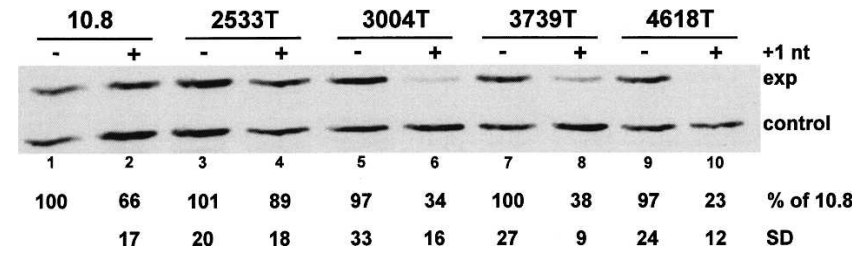

FIGURE 2. PTCs in pol have different effects on RNA level. RNase protection assay of RNA harvested from transiently transfected CEFs analyzed with a probe that detects the gag gene of RSV. The top band is the protected fragment corresponding to the unspliced viral RNA (exp). The bottom band is a stable viral loading control that gives a different size protected fragment using the same probe (LeBlanc and Beemon 2004). Quantification of bands was carried out by PhosphorImager analysis. A ratio was determined between the experimental and the control band in each lane, and normalized to 10.8, which was set at $100 \%$. Standard deviations (SD) are noted. Values represent the average of at least four experiments.

RNA led to production of only the Gag-Pol polyprotein, $\operatorname{Pr} 180^{\text {gag-pol }}$ (Fig. 1B, lane 3). As predicted, truncated readthrough protein products (denoted by an $*$ ) of approximately 78,123 , and $154 \mathrm{kDa}$ were observed when a PTC was placed within the pol gene at nt 2533,3739, and 4618, respectively (Fig. 1B, lanes $4-9$ ).

We next assayed the relative levels of unspliced RNA transcribed from each of the constructs bearing a PTC in pol by an RNase protection assay (Fig. 2). We co-transfected a stable viral loading control, and then probed the sample using a viral gag probe that can distinguish between the two viral RNAs (LeBlanc and Beemon 2004). We found that the $10.8+1$ transcript, which terminates translation at the end of pol, only accumulated $66 \%$ as much RNA as the 10.8 wild-type construct (Fig. 2, lanes 1,2). Interestingly, RNA bearing a termination codon at nt 2533, $\sim 50 \mathrm{nt}$ downstream of the gag natural termination codon, accumulated at wild-type levels in both the 10.8 and the $10.8+1$ constructs (Fig. 2, lanes 3,4). In contrast, termination codons placed further downstream, at nt 3004, 3739, and 4618 in the pol gene, led to RNA accumulation at the wild-type level in the 10.8 background (Fig. 2, lanes 5,7,9). However, only $23 \%-38 \%$ as much RNA as wild type accumulated in the $10.8+1$ background (Fig. 2, lanes 6,8,10). This experiment suggests that PTCs in pol that are infrequently translated do not induce NMD. However, when readthrough from gag is efficient, these same PTCs now cause a lower level of the unspliced RNA. The observation that RNA bearing a PTC just downstream of the gag natural termination codon still accumulated to high levels in the $10.8+1$ context (Fig. 2, lane 4 ) led us to examine this region more closely.

\section{A deletion of $400 \mathrm{nt}$ immediately after the gag termination codon elicits NMD}

Previous data indicate that various deletions downstream of the gag-pol junction lead to lower levels of unspliced viral RNA (Barker and Beemon 1994). The data presented above led us to propose that this region may be directing proper gag translation termination and stabilization of the RNA. RNA with a termination codon $\sim 50 \mathrm{nt}$ downstream of the gag natural termination codon accumulated at wild type levels, even when translated $100 \%$ of the time (Fig. 2, lane 4). When the termination codon was moved $500 \mathrm{nt}$ or more downstream in the same background, there was much less steady state RNA (Fig. 2, lanes 6,8,10).

We tested this hypothesis by deleting the putative RSV stability element (RSE) (nt 2486-2886) immediately after the natural termination codon in the 10.8 context, to generate the construct $\triangle$ RSE (Fig. 3A). After transient transfection in CEFs, the amount of viral RNA generated was assayed by RNase protection and found to be about fourfold less abundant than wild-type 10.8 RNA (Fig. 3B). This reduced level of viral RNA was similar to that seen previously with viral
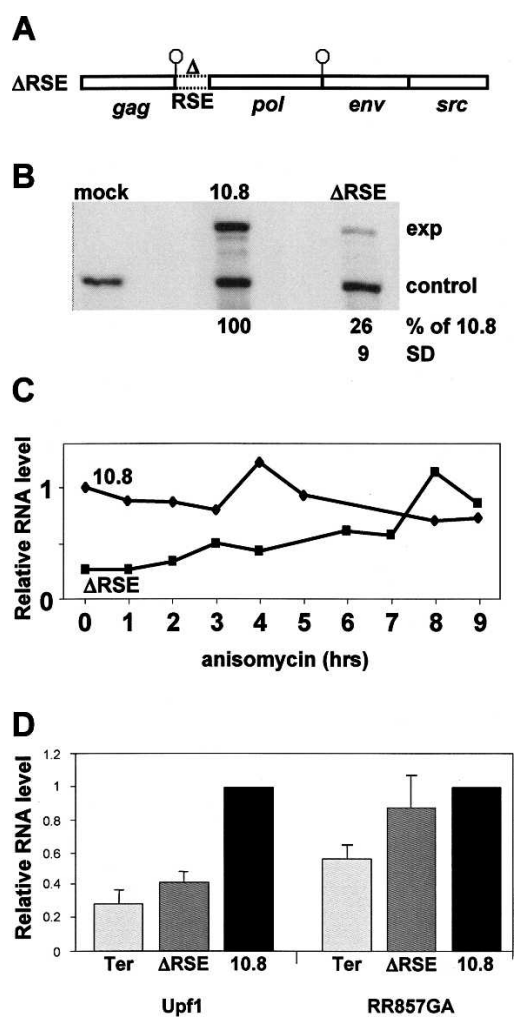

FIGURE 3. Deletion of the RSE causes NMD of the unspliced RNA. (A) Construct $\triangle$ RSE contains a deletion of the first 401 nt of the gag $3^{\prime}$ UTR, termed the "RSE" (nt 2486-2886) in the 10.8 viral clone. (B) A representative RNase protection assay of unspliced RNA derived from this deletion construct. The same loading control and probe as in Figure 2 were used, and the values are the average of at least five experiments, normalized and quantitated as in Figure 2. (C) CEFs were transiently transfected with the 10.8 and $\triangle$ RSE constructs, and RNA was harvested from 1 to $9 \mathrm{~h}$ after addition of $25 \mu \mathrm{g} / \mathrm{mL}$ of the translation inhibitor anisomycin (Calbiochem). RNA levels were assayed as in Figure 2 and normalized to the average values from $B$. (D) RNA from both the $\Delta$ RSE construct and a construct bearing a termination codon at nt 1924 in gag (Ter) were stabilized compared with wild-type constructs in the presence of a dominant negative Upf1 mutant (RR857GA). The average of three experiments is shown, normalized and quantitated as in Figure 2. 
RNAs containing a termination codon in gag (LeBlanc and Beemon 2004). The previous observation that RNAs with PTCs in the last $150 \mathrm{nt}$ of the gag gene, near the RSE, are increasingly more abundant than RNAs with PTCs in the rest of the gag gene should be noted (Barker and Beemon 1994).

To determine if the decrease in unspliced RNA with the $\triangle$ RSE construct was due to the NMD pathway, we tested its dependence on translation and the protein Upf1, both previously shown to be required for NMD (Leeds et al. 1991; Belgrader et al. 1993; Carter et al. 1995). Translation was inhibited by addition of anisomycin. Over a 9-h period, we saw up to a fourfold increase in the RNA level of the $\triangle \mathrm{RSE}$ construct (Fig. 3C). The level of 10.8 RNA was fairly uniform over this same time period, decreasing to $\sim 75 \%$ of its original level after $9 \mathrm{~h}$ (Fig. 3C).

We also blocked NMD by co-transfection of a dominant negative mutant of hUpf1, RR857GA (Mendell et al. 2002). When the Upfl mutant was co-transfected with $\triangle$ RSE, we saw a twofold increase in the unspliced RNA level, normalized to 10.8 RNA in each experiment (Fig. 3D). In the same experiment, we saw a similar increase in the amount of a viral RNA bearing a PTC at nt 1924 (Ter) (Barker and Beemon 1994). Co-transfection with either wild-type Upf1 or RR857GA caused no significant change in the level of the control 10.8 RNA to which the mutants are standardized. We conclude that deletion of this $3^{\prime}$ UTR region, the RSE, causes the natural termination codon to behave like a PTC.

\section{The RSE can stabilize a PTC in gag}

Since the wild-type RNA has the RSE downstream of the natural termination codon, we examined the effect of placing the RSE downstream of a PTC in the gag gene. To test this, we used the Ter construct with a PTC at nt 1924 in gag (Fig. 4A; Barker and Beemon 1994). The unspliced RNA from this PTC-containing RNA accumulated at $19 \%$ of wildtype RNA levels (Fig. 4A, lane 1). We then made a deletion in the gag gene, immediately downstream of the PTC insertion, extending to the natural termination codon. This deletion effectively positioned the RSE immediately downstream of the PTC. RNA from this construct accumulated to $\sim 62 \%$ of the wild-type level (Fig. 4A, lane 4). Subsequent deletion of the RSE from this construct resulted in decreased RNA levels (27\% of wild type) (Fig. $4 \mathrm{~A}$, lane 5), further implicating the RSE in stabilization.
Another interpretation of these results is that we were deleting some destabilizing element in the coding region. To address this issue, we first moved sense (forward) or anti-sense (reverse) forms of the 401-nt RSE into the gag coding region, immediately after a termination codon at nt 1250 (Fig. 4B, Ter for/rev $\Delta$ RSE). We observed approximately wild-type levels of unspliced RNA with the forward, but not the reverse insertion, suggesting that this element can stabilize the RNA with a termination codon at nt 1250 (Fig. 4B lanes 6,7). To ensure that the RNA containing only the reverse copy of the RSE was not less abundant simply because it now has no sense copy of the RSE, we left the natural copy of the RSE in place $(\sim 1200 \mathrm{nt}$ downstream of the PTC) and inserted another copy of the RSE after the PTC at nt 1250 (Ter for/rev). We again observed sense orientation-specific accumulation of the unspliced RNA (Fig. 4B, lanes 3,4). Since the RSE had this effect when it was found immediately downstream of a PTC, but not 1200 nt away, we conclude that the RSE acts on an immediately preceding translation termination codon.

To ask whether the observed decrease in RNA level was due to RNA instability, we used the drug actinomycin D to inhibit bulk cellular transcription in CEFs transiently transfected with 10.8, Ter (1924) (Fig. 4A), Ter for $\Delta$ RSE, and
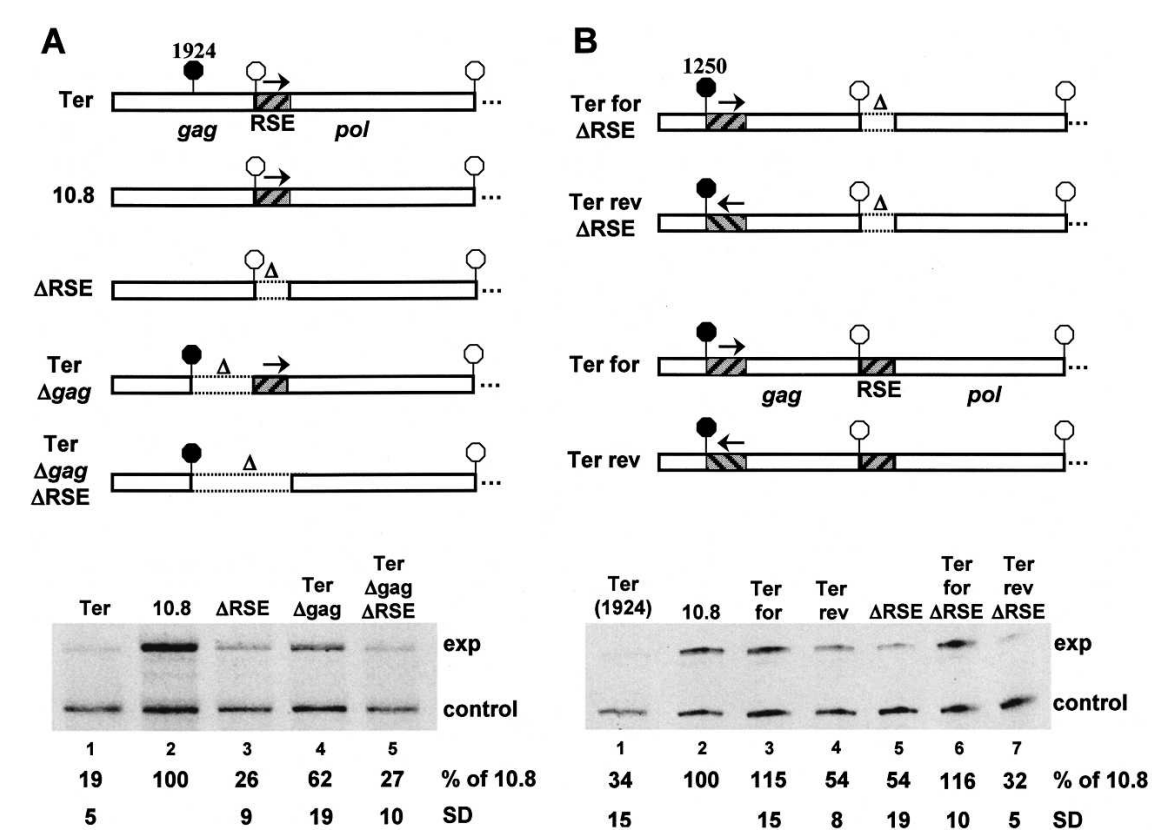

FIGURE 4. Movement of the RSE next to a premature termination codon increases RNA levels. $(A)$ Constructs were created to examine the effect of the RSE on PTC-containing RNAs. A partial deletion of the gag coding region (Ter $\Delta g a g$ ) positioned the RSE immediately $3^{\prime}$ of a PTC at nt 1924. In this diagram the RSE is indicated by a hatched box. A representative RNase protection assay is shown. Relative RNA levels were normalized and quantitated as in Figure 2, and the average of five experiments is shown. (B) Forward and reverse RSE fragments (forward and reverse hatched boxes) were inserted downstream of a PTC at nt 1250 . Constructs were tested both with and without the normal RSE downstream of the gag termination codon. A representative RNase protection assay is shown, with quantification from at least four experiments, normalized and quantified as in Figure 2. 
A

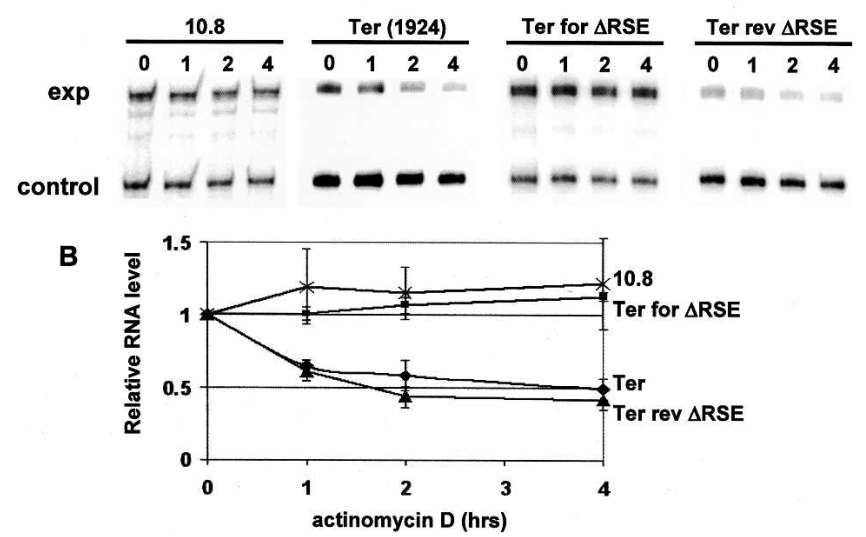

FIGURE 5. PTC-bearing RNA is stabilized by the RSE in the sense orientation. CEFs were transiently transfected with 10.8 , Ter (Fig. 4A), Ter for $\Delta$ RSE and Ter rev $\Delta$ RSE (Fig. $4 \mathrm{~B}$ ) constructs. Two days posttransfection the cells were treated with $2 \mu \mathrm{g} / \mathrm{mL}$ actinomycin $\mathrm{D}$, and RNA was harvested at 0-, 1-, 2-, and 4-h time points. Relative RNA levels were determined by RNase protection assay. A representative experiment is shown $(A)$, and the relative stabilities from five experiments, normalized and quantified as in Figure 2, are plotted $(B)$.

Ter rev $\triangle$ RSE (Fig. 4B). We then harvested RNA at 0-, 1-, 2-, and 4-h time points after addition of the drug. Representative gels are shown in Figure 5A. RNA levels at each time point were quantitated and plotted in Figure 5B, relative to the RNA level of each construct at zero time. We found that RNA bearing a PTC in gag followed by the RSE in the sense orientation (Ter for $\triangle \mathrm{RSE}$ ) was as stable as wild-type RNA. However, RNAs bearing the PTC alone (Ter) or with a PTC followed by the RSE in the reverse orientation (Ter rev $\Delta$ RSE) were both much less stable, with the RNA level dropping off rapidly during the first hour, and then stabilizing between 2 and $4 \mathrm{~h}$. From this result we can conclude that the RSE is affecting RNA abundance at the level of RNA stability.

\section{The RSE is a complex element}

After observing that insertion of the 401-nt RSE after a PTC was sufficient to stabilize the RNA (Fig. 5), we asked whether the entire RSE region was necessary for this. To address this, we made truncations from the $3^{\prime}$ end (construct $\mathrm{A}$ ), the $5^{\prime}$ end (construct $\mathrm{C}$ ), and both ends of the RSE (constructs B and D) (Fig. 6A). These different truncated fragments were then inserted immediately downstream of a PTC at nt 1250 in the gag gene. We found that a sense insertion of the last $280 \mathrm{nt}$ (C fragment) was sufficient to stabilize the RNA to $68 \%$ of the level of the fulllength RSE insertion (Fig. 6B, lane 9), while the anti-sense insertion at the same site led to only $27 \%$ of full length (Fig. $6 \mathrm{~B}$, lane 10). An RNA bearing an insertion of the first $250 \mathrm{nt}$ of the RSE (A fragment) was almost as stable as the fulllength insertion $(87 \%)$, but not significantly more stable than the same insertion in the reverse orientation (55\%) (Fig. 6B, lanes 5,6). It is possible that the reverse orientation of the A fragment also has some stabilizing ability. Interestingly, fragment $\mathrm{B}$, the 140-nt common region shared by fragments $\mathrm{A}$ and $\mathrm{C}$, was unable to stabilize the RNA to any extent (Fig. 6B, lane 7). A similar result was seen with fragment D (Fig. 6B, lane 11). The experiments with the truncated RSE fragments suggest that the element we are striving to characterize is complex and may have necessary, possibly redundant, features at both the $5^{\prime}$ and $3^{\prime}$ ends.

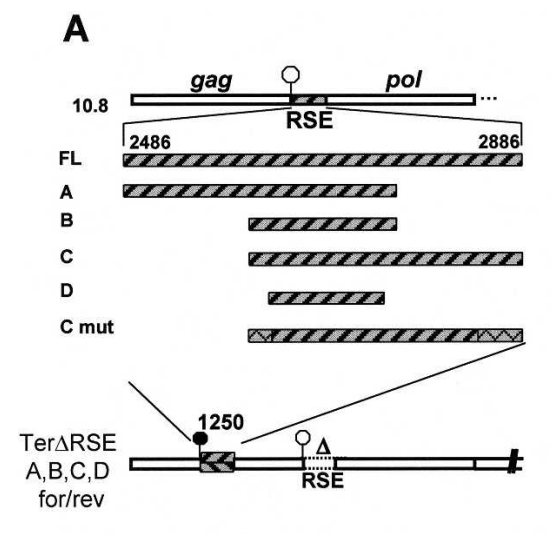

B
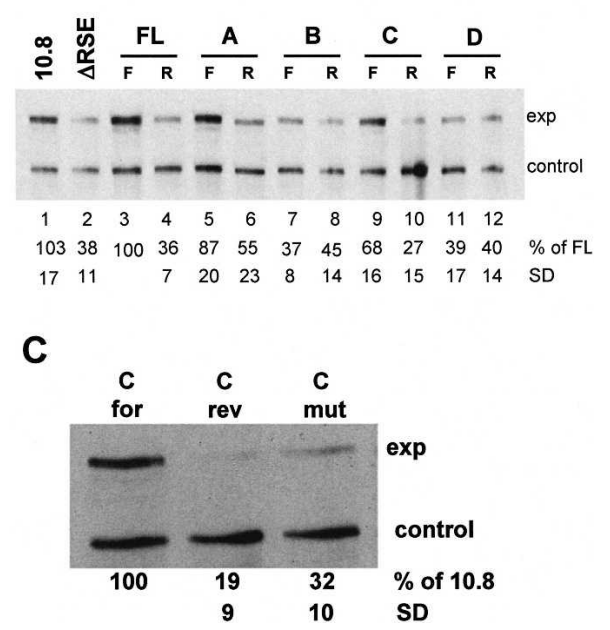

FIGURE 6. Two sub-fragments of the RSE can stabilize RNA. (A) To determine the minimal region that can stabilize a termination codon, various deletions were made at the ends of the RSE. Additionally, three groups of point mutations ( $14 \mathrm{nt}$ mutated) were made in putative single stranded regions near the ends of the $\mathrm{C}$ fragment (C Mut). One group of five point mutations is located at the $5^{\prime}$ end. One group of five and one group of four nt were mutated at the $3^{\prime}$ end. These sub-fragments were inserted after a PTC at nt 1250. (FL) Full length. (B) A representative RNase protection assay is shown with average values from four experiments, and normalized to full-length insertion. (F) Forward; (R) reverse orientations of the RSE fragment. $(C)$ The mutated fragment $\mathrm{C}$ of the RSE was inserted immediately after a PTC at nt 1250 in the $\triangle$ RSE construct. RNase protection assays were used to determine relative RNA levels. These were compared to those of constructs bearing the wild-type $C$ fragment in the forward and reverse orientations. Average values from six experiments are indicated. 
Although clearly not sufficient, the common region is thought to be necessary based on previous deletions in this area (Barker and Beemon 1994).

Since fragment $\mathrm{C}$ from Figure 6A induced the greatest stabilization relative to its insertion in the reverse orientation, we decided to use this fragment in future studies. A group of five point mutations was generated near the $5^{\prime}$ end, and nine point mutations near the $3^{\prime}$ end in regions that were predicted to be single stranded by MFOLD (Zuker 2003; data not shown). When this mutated fragment ( $\mathrm{C}$ mut) was inserted after a termination codon at nt 1250 in the gag coding region, the resulting unspliced RNA was $\sim 32 \%$ as abundant as the "C for" RNA (Fig. 6C). To further dissect the region containing these point mutations, we made constructs with mutations at only the $5^{\prime}$ or the $3^{\prime}$ end. Although both single end mutations had an intermediate effect, the $3^{\prime}$ end appeared to be more crucial for accumulation of RNA (data not shown). This is consistent with the observation that the region shared by $\mathrm{A}$ and $\mathrm{C}$ is insufficient to promote a high level of RNA accumulation. We conclude that the RSE may have a necessary but not sufficient middle region, and a necessary but redundant feature at each end.

\section{DISCUSSION}

In this report we examined the mechanism by which termination codons are distinguished as natural or premature in RSV unspliced RNA expressed in chicken cells. Surprisingly, we observed that a 400-nt deletion downstream of the gag termination codon resulted in its recognition as a PTC, leading to NMD. When this 3' UTR region (which we call the RSE) was inserted downstream of a PTC in the gag gene, the RNA was stabilized. The anti-sense orientation, or a mutated RSE sequence, did not stabilize the RNA. These findings differ from previous observations in higher eukaryotic cells showing that unspliced RNA does not undergo NMD and that a downstream EJC triggers NMD (Le Hir et al. 2000a; Lykke-Andersen et al. 2000; Maquat and Li 2001; Brocke et al. 2002). We hypothesize that, in chicken cells, the RSE promotes proper translation termination of the viral RNA when next to a termination codon, and in its absence, NMD occurs. The RSE may recruit stabilization factors such as the eukaryotic release factors (eRFs), the Poly(A) binding protein, or cap binding proteins, in order to overcome such a long $3^{\prime}$ UTR. In yeast, tethering of either the Poly(A) binding protein or eRF3 downstream of a coding region PTC stabilized the RNA (Amrani et al. 2004).

\section{Different organisms have developed alternative mechanisms of NMD}

NMD was first observed in yeast (Losson and Lacroute 1979), where it requires both translation and the Upf proteins, just as it does in eukaryotic cells. In yeast, only about $4 \%$ of genes are spliced (Lopez and Seraphin 2000), and the identification of
PTCs does not require splicing (Gonzalez et al. 2001). Recent work in yeast suggests that this organism differentiates premature from normal termination events by a faux UTR model (Amrani et al. 2004). This model posits that the natural termination codon is identified by its proximity to an appropriate $3^{\prime}$ UTR and termination stabilizing factors. Conversely, the region downstream of a PTC within the coding region is a "faux" (or false) UTR, which is unfit to direct proper termination (Hilleren and Parker 1999). Jacobson and coworkers (Amrani et al. 2004) have shown that translation termination at a PTC is aberrant, and the ribosome does not properly release from the RNA. A natural 3' UTR can promote proper termination, as can tethered poly(A) binding protein. This challenges a previous model in yeast that suggests the existence of a cis-acting exonic downstream sequence element (DSE) (Ruiz-Echevarria et al. 1998; Gonzalez et al. 2000).

Some notable exceptions to the generally accepted NMD model exist (Enssle et al. 1993; Barker and Beemon 1994; Carter et al. 1996; Zhang et al. 1998b; Gudikote and Wilkinson 2002; Wang et al. 2002; LeBlanc and Beemon 2004). In mammalian cells, a mark deposited by a prior splicing event downstream of a termination codon appears to signal premature termination. Thus, an RNA without an intron downstream of a PTC should be immune to NMD (Maquat and Li 2001; Brocke et al. 2002). However, a loosely characterized "failsafe signal" in globin mRNA acts to direct NMD even in the absence of a downstream intron (Zhang et al. 1998b). Similarly, we have observed that a viral mRNA undergoes NMD in chicken cells in the absence of splicing, thus it does not conform to the rules established for NMD in mammalian and, presumably, other higher eukaryotic cells. Izaurralde and coworkers (Gatfield et al. 2003) first highlighted differences between mammals and other metazoans by showing that knockdown of several key EJC components had no effect on NMD, and PTC discrimination appears to be independent of exon-exon boundaries in Drosophila. Our observations are similar to this work in Drosophila in that we observe splicingindependent NMD in chicken cells. Like mammals, chickens utilize extensive mRNA splicing (Hillier et al. 2004).

\section{Possible NMD mechanisms for RSV RNA}

We have observed that a PTC in the pol gene elicits decay only when translated efficiently, suggesting that PTC recognition during any round of translation does not induce NMD. Similarly, it has been proposed that in mammalian cells, NMD occurs only during a pioneer round of translation (Lejeune et al. 2004). In contrast, studies in both yeast and rats have shown a decrease in RNA levels due to an infrequently translated PTC (Buzina and Shulman 1999; Plant et al. 2004). This is not surprising since RNAs in yeast can undergo NMD during any round of translation (Maderazo et al. 2003). Further, the RNA tested in rats was Ig- $\mu$ (Buzina and Shulman 1999), a transcript that seems to require special NMD rules (Buhler et al. 2004, 2005). 
Similar to experiments in yeast where a $3^{\prime}$ UTR was used to stabilize a PTC (Amrani et al. 2004), we used various deletions and insertions to position the RSE immediately downstream of a PTC in the gag gene. In all cases, the RSE showed sequence-specific stabilization of the RNA. In yeast, poly(A) binding protein tethered downstream of a PTC caused stabilization. It therefore seems plausible that a feature of the correct $3^{\prime}$ UTR may involve proximity to the $\operatorname{poly}(\mathrm{A})$ tail or its associated factors such as the eRFs. However, when we moved the proper gag $3^{\prime}$ UTR (the RSE) immediately after a PTC, the poly(A) tail was still about 7 $\mathrm{kb}$ away. It is conceivable that the RSE itself interacts with factors that recruit the eRFs. Alternatively, this region may help the viral unspliced RNA overcome an extremely long 3' UTR, possibly by promoting long-range interactions with distant parts of the transcript. Without this stability element, the untranslated region following a termination codon is seen as aberrant by the cell and undergoes decay.

An alternative hypothesis is that there is a DSE in the coding region of RSV, as proposed in yeast (Zhang et al. 1995). A DSE-like sequence might be found in the RSV coding region, potentially binding EJC proteins, SR proteins, or other potential RNA decay triggering factors such as the recently identified protein Staufen 1 (LykkeAndersen et al. 2000; Zhang and Krainer 2004; Kim et al. 2005). Also, it is arguable that the deletion analysis we carried out (Fig. 4A) may be changing the distance between the termination codon and a downstream DSE. However, we observed stabilization of the PTC- bearing RNA only by a sense insertion of the RSE (Fig. 4B). If we were simply moving a DSE too far away, then the antisense insertion would be predicted to stabilize the RNA in the same fashion. Thus, it appears that the stabilization is due to specific sequences within the RSE, which may recruit positive stability factors or repel negative decay factors.

\section{Conserved 3' UTR motifs may play a role in stabilizing natural termination events}

A recent report aligned untranslated portions of many annotated genes from human, rat, mouse, and dog (Xie et al. 2005). Several conserved motifs were found in both the promoter regions of genes and the $3^{\prime}$ UTRs of these genes. In addition to an enriched pool of predicted eight-mer miRNA target sites, conserved motifs were found in the $3^{\prime}$ UTR. Although several of these motifs have a known function (poly(A) site, AU rich elements), most of the 3' UTR motifs have no identified function. Perhaps some of these unknown motifs are involved in stabilization of a natural termination codon. The RSE sequence contains similarities to some of the $3^{\prime}$ UTR motifs, which overlap our $3^{\prime}$ set of mutations. Future experiments will be designed to dissect the region containing these point mutations and to ask whether they disrupt common termination-stabilizing motifs.

\section{Implications}

A recent report describes up-regulation of human endogenous retroviral RNAs when Upf1 is knocked down by RNAi in human cells (Mendell et al. 2004). It appears that the NMD pathway may be keeping these viral RNAs in check, even though most of the coding regions lack downstream introns (Bohne et al. 2005). The observation that inactivation of NMD affects transcripts from human endogenous retroviruses suggests that they may undergo NMD in human cells in a manner similar to the decay of avian retroviral transcripts in chicken cells.

Perhaps as higher organisms evolved the formation of an EJC, this became the primary signal that a termination codon was a PTC. Chicken and Drosophila, although possessing EJC protein genes, may not require the EJC to promote NMD. Thus far all of the work probing the composition of the EJC has been done in human cells. These other organisms may be relying on an ancient, alternate mechanism to direct NMD. It has recently been suggested that different organisms have evolved mechanisms of PTC determination best suited for the amount and type of pre-mRNA processing that each one utilizes (Tange et al. 2004; Conti and Izaurralde 2005; Lejeune and Maquat 2005). Our identification of the cis-acting RSE sequence helps to close the gap in the understanding of how NMD works in different organisms. Due to its important role in viral replication, the full-length RSV RNA is unspliced, exported from the nucleus, and translated, while bearing a 7$\mathrm{kb} 3^{\prime}$ UTR. The RSE may have evolved in order to help the virus overcome the RNA destabilization from such a long $3^{\prime}$ UTR. Understanding how this works may help us realize why transcripts with extended $3^{\prime}$ UTRs are NMD substrates (Hilleren and Parker 1999) and eventually develop a better understanding of the mechanism of NMD.

\section{MATERIALS AND METHODS}

\section{Plasmid construction}

The 10.8 plasmid is a permuted copy of the Prague C strain of RSV (Accession number: NC_001407), containing a deletion in the nucleocapsid region of the gag gene (Meric et al. 1988). The 10.8 +1 construct was generated with the Quickchange site directed mutagenesis kit (Statagene), using the primer 5'-GGGCTCCGCTT GACAAATTTAATAGGGAGGGCC-3', and its reverse complement. The underlined A is the inserted nt, immediately upstream of the gag termination codon. The $10.8 \mathrm{pol}$ Ter and $10.8+1$ pol Ter constructs were also made by Quickchange, generating the following point mutations which changed normal codons into termination codons: A2533T, T3005A, A3739T, and G4620A.

The $\Delta$ RSE construct contains a deletion of nt 2486-2886. This was generated by inverse PCR from 10.8, using the following primers; $\Delta 401 \mathrm{~F}-5^{\prime}$-CTCCCCTCTGTGAATAACCAGGCCCCC GC- $3^{\prime}$ and $\Delta 401 \mathrm{R}-5^{\prime}$-CTATAAATTTGTCAAGCGGAGCCCTA GCC- $3^{\prime}$. The primers were kinased using T4 polynucleotide kinase (NEB) and used in a touchdown PCR reaction.

The Ter $\Delta$ gag construct was created using the plasmid 1924T described previously (Barker and Beemon 1994). 1924T contains a 
linker, with stop codons in all three reading frames, inserted at the SmaI site at nt 1924. Nucleotides 1934 (immediately after the in frame PTC) through 2485 (up to and including the gag stop codon) were deleted by inverse PCR as described above, using the following primers: 2486for - 5'-GGGAGGGCCACTGTTCTCCTGTTGCGC-3', and KTreverse - $5^{\prime}$-CTAGACTAGCGGGGATCCACAAGTGTAGCAG AGCCC- $3^{\prime}$. Construct Ter $\Delta$ gag $\Delta$ RSE was made by inverse PCR using primers KTreverse and $\Delta 401 \mathrm{~F}$ described above. This construct has a deletion extending from nt 1934-2887. Construct 1547C (loading control) is a similar construct with a control insertion (no PTCs) at nt 1547 (Barker and Beemon 1994). The different sites of linker insertion led to two different products upon RNase digestion of the complementary riboprobe (LeBlanc and Beemon 2004).

To make the constructs Ter for/rev, the RSE sequence (nt 24832887) was amplified by PCR from the 10.8 plasmid template, using primers possessing AatII sites at their $5^{\prime}$ ends. These PCR fragments were cloned into the unique AatII site at nt 1250 in both the forward and reverse orientations. The natural gag termination codon is present at the $5^{\prime}$ end of the RSE forward fragment. A termination codon was also introduced at the $5^{\prime}$ end of the RSE in the reverse orietation. Ter $\Delta$ RSE for/rev were made in the same fashion and cloned into the $\Delta$ RSE background. Fragments A, B, C, or D were made by PCR, and inserted at the same site. Each fragment begins with a TAG stop codon and then encompasses the following sequences: (A) 2486-2735, (B) 2600-2735, (C) 2600-2880, (D) 2617-2706. Note, fragment C ends 7 nt before the end of the full-length RSE.

The construct $\mathrm{C}$ mutant contains the same insertion as $\mathrm{C}$ above, with a series of point mutations. The mutagenic primers used to make this fragment are: MutCfor 5'-GCGCGGACGTCTAGCGC TAACGCAATTAGTGGAAAAAGATAAAGCGTTAGGACATATAG AACC-3', and MutCrev 5'-GCGGACGTCTAGTAATAGGTCAA CACCTTGAGGTCTAAG-3', with the underlined nt mutated from the wild-type sequence. The $3^{\prime}$ end of this fragment ends at the same nt as the $3^{\prime}$ end of the full-length RSE.

\section{RNA isolation and RNase protection assays}

In vitro transcription of the RSV gag probe was carried out as described in LeBlanc and Beemon (2004). RNA isolation and RNase protection assays were done as described previously (LeBlanc and Beemon 2004). In brief, total cellular RNA was harvested $40-48 \mathrm{~h}$ after transfection, using RNA-Bee (Tel-Test) per the manufacturer's instructions. After hybridization with 250,000 cpm of probe, RNA was digested with $10 \mathrm{U} / \mathrm{mL}$ RNase T1 (Calbiochem) and $5 \mu \mathrm{g} / \mathrm{mL}$ RNase $\mathrm{A}$ at $34^{\circ} \mathrm{C}$. After $45 \mathrm{~min}$ the digestion was stopped by addition of sodium dodecyl sulfate and Proteinase $\mathrm{K}$ (Roche) and incubated at $37^{\circ} \mathrm{C}$ for $15 \mathrm{~min}$. RNA was extracted with phenol:chloroform:isoamyl alcohol, precipitated with ethanol, and resuspended in $8 \mathrm{M}$ urea loading buffer, and denatured for $5 \mathrm{~min}$ at $95^{\circ} \mathrm{C}$. Samples were loaded onto a $6 \%$ acrylamide, $8 \mathrm{M}$ urea gel, and electrophoresed at $60 \mathrm{~W}$ for 2-4 h. RNA levels were quantified using either an Instant Imager (Packard), or a Typhoon 9410 PhosphorImager (Amersham).

\section{Cell culture and transfection}

Cell culture and transfection of secondary CEF cells were carried out as described in LeBlanc and Beemon (2004). Transfections were carried out in $60-\mathrm{mm}$ plates that were $90 \%-95 \%$ confluent. Aniso- mycin (Calbiochem) was used at a final concentration of $25 \mu \mathrm{g} / \mathrm{mL}$ to inhibit cellular translation. Actinomycin D (Calbiochem) was used at a final concentration of $2 \mu \mathrm{g} / \mathrm{mL}$ to inhibit cellular transcription.

\section{Western blot analysis}

$\mathrm{CEF}$ cells were removed from the plates at $39^{\circ} \mathrm{C}$ with $0.05 \%$ trypsinEDTA (Gibco) and pelleted. Western blotting was carried out as described in LeBlanc and Beemon (2004). RSV Gag and Gag-Pol proteins were detected using polyclonal rabbit anti-avian myelobastosis virus (AMV) p19 gag serum, obtained from D.P. Bolognesi (Duke University), and ImmunoPure goat anti-rabbit Immunoglobulin G (heavy plus light chains, horseradish peroxidase conjugated; Pierce). Blots were then visualized by autoradiography using the enhanced chemiluminescence reagent kit (Amersham).

\section{ACKNOWLEDGMENTS}

This work was supported by NIH grant R01 CA48746 to K.L.B. J.E.W. was supported in part by NIH predoctoral training grant T32GM07231. We thank D.P. Bolognesi for the anti-p19 antibody and Hal Dietz for the hUpf1 and RR857GA plasmids and helpful discussions. We also thank Jason LeBlanc and Rena Xian for reviews of the manuscript and Yingying Li for technical assistance.

Received June 2, 2005; accepted October 10, 2005.

\section{REFERENCES}

Amrani, N., Ganesan, R., Kervestin, S., Mangus, D.A., Ghosh, S., and Jacobson, A. 2004. A faux 3'-UTR promotes aberrant termination and triggers nonsense-mediated mRNA decay. Nature 432: 112-118.

Barker, G.F. and Beemon, K. 1991. Nonsense codons within the Rous sarcoma virus gag gene decrease the stability of unspliced viral RNA. Mol. Cell. Biol. 11: 2760-2768.

. 1994. Rous sarcoma virus RNA stability requires an open reading frame in the gag gene and sequences downstream of the gag-pol junction. Mol. Cell. Biol. 14: 1986-1996.

Belgrader, P., Cheng, J., and Maquat, L.E. 1993. Evidence to implicate translation by ribosomes in the mechanism by which nonsense codons reduce the nuclear level of human triosephosphate isomerase mRNA. Proc. Natl. Acad. Sci. 90: 482-486.

Bohne, J., Wodrich, H., and Krausslich, H.G. 2005. Splicing of human immunodeficiency virus RNA is position-dependent suggesting sequential removal of introns from the $5^{\prime}$ end. Nucleic Acids Res. 33: $825-837$.

Brocke, K.S., Neu-Yilik, G., Gehring, N.H., Hentze, M.W., and Kulozik, A.E. 2002. The human intronless melanocortin 4-receptor gene is NMD insensitive. Hum. Mol. Genet. 11: 331-335.

Buhler, M., Paillusson, A., and Muhlemann, O. 2004. Efficient downregulation of immunoglobulin mu mRNA with premature translation-termination codons requires the $5^{\prime}$-half of the VDJ exon. Nucleic Acids Res. 32: 3304-3315.

Buhler, M., Mohn, F., Stalder, L., and Muhlemann, O. 2005. Transcriptional silencing of nonsense codon-containing immunoglobulin minigenes. Mol. Cell 18: 307-317.

Buzina, A. and Shulman, M.J. 1999. Infrequent translation of a nonsense codon is sufficient to decrease mRNA level. Mol. Biol. Cell 10: 515-524.

Carter, M.S., Doskow, J., Morris, P., Li, S., Nhim, R.P., Sandstedt, S., and Wilkinson, M.F. 1995. A regulatory mechanism that detects premature nonsense codons in T-cell receptor transcripts in vivo is reversed by protein synthesis inhibitors in vitro. J. Biol. Chem. 270: 28995-29003. 
Carter, M.S., Li, S., and Wilkinson, M.F. 1996. A splicing-dependent regulatory mechanism that detects translation signals. EMBO J. 15: 5965-5975.

Chan, C.C., Dostie, J., Diem, M.D., Feng, W., Mann, M., Rappsilber, J., and Dreyfuss, G. 2004. eIF4A3 is a novel component of the exon junction complex. RNA 10: 200-209.

Conti, E. and Izaurralde, E. 2005. Nonsense-mediated mRNA decay: Molecular insights and mechanistic variations across species. Curr. Opin. Cell Biol. 17: 316-325.

Enssle, J., Kugler, W., Hentze, M.W., and Kulozik, A.E. 1993. Determination of mRNA fate by different RNA polymerase II promoters. Proc. Natl. Acad. Sci. 90: 10091-10095.

Gatfield, D., Unterholzner, L., Ciccarelli, F.D., Bork, P., and Izaurralde, E. 2003. Nonsense-mediated mRNA decay in Drosophila: At the intersection of the yeast and mammalian pathways. EMBO J. 22: 3960-3970.

Gonzalez, C.I., Ruiz-Echevarria, M.J., Vasudevan, S., Henry, M.F., and Peltz, S.W. 2000. The yeast hnRNP-like protein Hrp1/Nab4 marks a transcript for nonsense-mediated mRNA decay. Mol. Cell 5: 489-499.

Gonzalez, C.I., Bhattacharya, A., Wang, W., and Peltz, S.W. 2001. Nonsense-mediated mRNA decay in Saccharomyces cerevisiae. Gene 274: 15-25.

Gudikote, J.P. and Wilkinson, M.F. 2002. T-cell receptor sequences that elicit strong down-regulation of premature termination codon-bearing transcripts. EMBO J. 21: 125-134.

He, F. and Jacobson, A. 2001. Upflp, Nmd2p, and Upf3p regulate the decapping and exonucleolytic degradation of both nonsense-containing mRNAs and wild-type mRNAs. Mol. Cell. Biol. 21: 1515-1530.

Hilleren, P. and Parker, R. 1999. Mechanisms of mRNA surveillance in eukaryotes. Annu. Rev. Genet. 33: 229-260.

Hillier, L.W., Miller, W., Birney, E., Warren, W., Hardison, R.C., Ponting, C.P., Bork, P., Burt, D.W., Groenen, M.A., Delany, M.E., et al. 2004. Sequence and comparative analysis of the chicken genome provide unique perspectives on vertebrate evolution. Nature 432: 695-716.

Kim, V.N., Kataoka, N., and Dreyfuss, G. 2001a. Role of the nonsensemediated decay factor hUpf3 in the splicing-dependent exon-exon junction complex. Science 293: 1832-1836.

Kim, V.N., Yong, J., Kataoka, N., Abel, L., Diem, M.D, and Dreyfuss, G. 2001b. The Y14 protein communicates to the cytoplasm the position of exon-exon junctions. EMBO J. 20: 2062-2068.

Kim, Y.K., Furic, L., Desgroseillers, L., and Maquat., L.E. 2005. Mammalian Staufen1 recruits Upf1 to specific mRNA 3'UTRs so as to elicit mRNA decay. Cell 120: 195-208.

Le Hir, H., Izaurralde, E., Maquat, L.E., and Moore, M.J. 2000a. The spliceosome deposits multiple proteins 20-24 nucleotides upstream of mRNA exon-exon junctions. EMBO J. 19: 6860-6869.

Le Hir, H., Moore, M.J., and Maquat, L.E. 2000b. Pre-mRNA splicing alters mRNP composition: Evidence for stable association of proteins at exon-exon junctions. Genes \& Dev. 14: 1098-1108.

Le Hir, H., Gatfield, D., Braun, I.C., Forler, D., and Izaurralde, E. 2001. The protein Mago provides a link between splicing and mRNA localization. EMBO Rep. 2: 1119-1124.

LeBlanc, J.J. and Beemon, K.L. 2004. Unspliced Rous sarcoma virus genomic RNAs are translated and subjected to nonsense-mediated mRNA decay before packaging. J. Virol. 78: 5139-5146.

Leeds, P., Peltz, S.W., Jacobson, A., and Culbertson, M.R. 1991. The product of the yeast UPF1 gene is required for rapid turnover of mRNAs containing a premature translational termination codon. Genes \& Dev. 5: 2303-2314.

Lejeune, F. and Maquat, L.E. 2005. Mechanistic links between nonsense-mediated mRNA decay and pre-mRNA splicing in mammalian cells. Curr. Opin. Cell Biol. 17: 309-315.

Lejeune, F., Ranganathan, A.C., and Maquat, L.E. 2004. eIF4G is required for the pioneer round of translation in mammalian cells. Nat. Struct. Mol. Biol. 11: 992-1000.

Lewis, B.P., Green, R.E., and Brenner, S.E. 2003. Evidence for the widespread coupling of alternative splicing and nonsense-mediated mRNA decay in humans. Proc. Natl. Acad. Sci. 100: 189-192.

Lopez, P.J. and Seraphin, B. 2000. YIDB: The Yeast Intron DataBase. Nucleic Acids Res. 28: 85-86.
Losson, R. and Lacroute, F. 1979. Interference of nonsense mutations with eukaryotic messenger RNA stability. Proc. Natl. Acad. Sci. 76: 5134-5137.

Lykke-Andersen, J. 2002. Identification of a human decapping complex associated with hUpf proteins in nonsense-mediated decay. Mol. Cell. Biol. 22: 8114-8121.

Lykke-Andersen, J., Shu, M.D., and Steitz, J.A. 2000. Human Upf proteins target an mRNA for nonsense-mediated decay when bound downstream of a termination codon. Cell 103: 1121-1131.

Maderazo, A.B., Belk, J.P., He, F., and Jacobson, A. 2003. Nonsensecontaining mRNAs that accumulate in the absence of a functional nonsense-mediated mRNA decay pathway are destabilized rapidly upon its restitution. Mol. Cell. Biol. 23: 842-851.

Maquat, L.E. 2004. Nonsense-mediated mRNA decay: Splicing, translation and mRNP dynamics. Nat. Rev. Mol. Cell Biol. 5: 89-99.

Maquat, L.E. and Li, X. 2001. Mammalian heat shock p70 and histone $\mathrm{H} 4$ transcripts, which derive from naturally intronless genes, are immune to nonsense-mediated decay. RNA 7: 445-456.

Mendell, J.T., ap Rhys, C.M., and Dietz, H.C. 2002. Separable roles for rent1/hUpfl in altered splicing and decay of nonsense transcripts. Science 298: 419-422.

Mendell, J.T., Sharifi, N.A., Meyers, J.L., Martinez-Murillo, F., and Dietz, H.C. 2004. Nonsense surveillance regulates expression of diverse classes of mammalian transcripts and mutes genomic noise. Nat. Genet. 36: 1073-1078.

Meric, C., Gouilloud, E., and Spahr, P.F. 1988. Mutations in Rous sarcoma virus nucleocapsid protein p12 (NC): Deletions of CysHis boxes. J. Virol. 62: 3328-3333.

Neu-Yilik, G., Gehring, N.H., Hentze, M.W., and Kulozik, A.E. 2004. Nonsense-mediated mRNA decay: From vacuum cleaner to Swiss army knife. Genome Biol. 5: 218.

Plant, E.P., Wang, P., Jacobs, J.L., and Dinman, J.D. 2004. A programmed -1 ribosomal frameshift signal can function as a cis-acting mRNA destabilizing element. Nucleic Acids Res. 32: 784-790.

Ruiz-Echevarria, M.J., Gonzalez, C.I., and Peltz, S.W. 1998. Identifying the right stop: Determining how the surveillance complex recognizes and degrades an aberrant mRNA. EMBO J. 17: 575-589.

Sun, X., Moriarty, P.M., and Maquat, L.E. 2000. Nonsense-mediated decay of glutathione peroxidase 1 mRNA in the cytoplasm depends on intron position. EMBO J. 19: 4734-4744.

Swanstrom, R. and Wills, J.W. 1997. Synthesis, assembly, and processing of viral proteins. In Retroviruses (eds. J.M. Coffin et al.), pp. 263-334. Cold Spring Harbor Laboratory Press, Cold Spring Harbor, NY.

Tange, T.O., Nott, A., and Moore, M.J. 2004. The ever-increasing complexities of the exon junction complex. Curr. Opin. Cell Biol. 16: 279-284.

Wang, J., Gudikote, J.P., Olivas, O.R., and Wilkinson, M.F. 2002. Boundary-independent polar nonsense-mediated decay. EMBO Rep. 3: 274-279.

Wilkinson, M.F. 2005. A new function for nonsense-mediated mRNAdecay factors. Trends Genet. 21: 143-148.

Xie, X., Lu, J., Kulbokas, E.J., Golub, T.R., Mootha, V., Lindblad-Toh, K., Lander, E.S., and Kellis, M. 2005. Systematic discovery of regulatory motifs in human promoters and $3^{\prime}$ UTRs by comparison of several mammals. Nature 434: 338-345.

Zhang, Z. and Krainer, A.R. 2004. Involvement of SR proteins in mRNA surveillance. Mol. Cell 16: 597-607.

Zhang, S., Ruiz-Echevarria, M.J., Quan, Y., and Peltz, S.W. 1995. Identification and characterization of a sequence motif involved in nonsensemediated mRNA decay. Mol. Cell. Biol. 15: 2231-2244.

Zhang, J., Sun, X., Qian, Y., LaDuca, J.P., and Maquat, L.E. 1998a. At least one intron is required for the nonsense-mediated decay of triosephosphate isomerase mRNA: A possible link between nuclear splicing and cytoplasmic translation. Mol. Cell. Biol. 18: 5272-5283.

Zhang, J., Sun, X., Qian, Y., and Maquat, L.E. 1998b. Intron function in the nonsense-mediated decay of $\beta$-globin mRNA: Indications that pre-mRNA splicing in the nucleus can influence mRNA translation in the cytoplasm. RNA 4: 801-815.

Zuker, M. 2003. Mfold web server for nucleic acid folding and hybridization prediction. Nucleic Acids Res. 31: 3406-3415. 

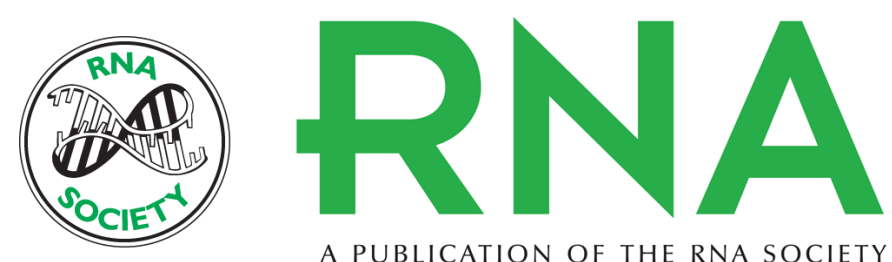

A PUBLICATION OF THE RNA SOCIETY

\section{A 3' UTR sequence stabilizes termination codons in the unspliced RNA of Rous sarcoma virus}

JASON E. WEIL and KAREN L. BEEMON

RNA 2006 12: 102-110

References This article cites 55 articles, 29 of which can be accessed free at: http://rnajournal.cshlp.org/content/12/1/102.full.html\#ref-list-1

\section{License}

Email Alerting Receive free email alerts when new articles cite this article - sign up in the box at the top Service right corner of the article or click here.

To subscribe to RNA go to:

http://rnajournal.cshlp.org/subscriptions 